\title{
Evaluación cardiorrespiratoria de conejos (Oryctolagus cuniculus) anestesiados con una combinación de tramadol, acepromacina, xilazina y ketamina
}

\author{
Cardiorespiratory evaluation of rabbits (Oryctolagus cuniculus) anesthetized \\ with a combination of tramadol, acepromazine, xylazine and ketamine
}

\author{
JJ Pérez-Rivero*, E Rendón-Franco
}

Universidad Autónoma Mertopolitana Unidad Xochimilco, Departamento de Producción Agrícola y Animal, México D.F.

\begin{abstract}
SUMMARY
Rabbits are considered as difficult animals in relation to anaesthesia due to their anatomical and physiological peculiarities. A mortality rate higher that $5 \%$ occurs during general anesthesia; furthermore, anaesthetic combinations that are rarely used include trans-surgical analgesia. Twenty-five clinically healthy New Zealand rabbits (Oryctolagus cuniculus) were anaesthetised with a combination of ketamine $(50 \mathrm{mg} / \mathrm{kg}), \mathrm{xilazine}(5 \mathrm{mg} / \mathrm{kg})$ and acepromazine $(0.5 \mathrm{mg} / \mathrm{kg})$ intramuscularly; 10 minutes later tramadol $(5 \mathrm{mg} / \mathrm{kg})$ was applied intravenously. Cardiac and respiratory rates and pulseoximetry were recorded before the administering anaesthesia at minute " 0 " and later every 10 minutes until minute 60 . The average cardiac rate was $154 \pm 28$ beats per minute and the average respiratory frequency was $63 \pm 33$ per minute during the procedure. Average pulse-oximetry was of $88 \pm 8 \%$ $(\mathrm{P}<0.05)$. Anaesthetic protocol with tramadol, acepromazine, xylazine and ketamine is safe during surgery training at the used doses, where FC, FR and Ox seem to be stable, however, oxygen supplementation is recommended during anaesthesia.
\end{abstract}

Key words: rabbit, anesthesia, tramadol, cardiorespiratory.

\section{RESUMEN}

Los conejos son considerados animales difíciles de anestesiar, debido a sus peculiaridades anatómicas y fisiológicas. Durante la anestesia general se presenta una mortalidad superior al 5\%, además las combinaciones anestésicas utilizadas pocas veces incluyen analgésicos de manera transoperatoria. 25 conejos Nueva Zelanda clínicamente sanos fueron anestesiados con la combinación de ketamina (50mg/kg), xilacina (5mg/kg), acepromacina (0,5 mg/ $\mathrm{kg}$ ), por vía intramuscular, 10 minutos después se aplicó tramadol $(5 \mathrm{mg} / \mathrm{kg})$ por vía endovenosa. Se evaluó frecuencia cardiaca, respiratoria y oximetría antes de la aplicación de la anestesia al minuto" "0" y posteriormente cada 10 minutos hasta el minuto 60. La frecuencia cardiaca y respiratoria tuvieron una diferencia significativa $(\mathrm{P}<0,05)$ entre el minuto " 0 " y el minuto "10" a partir de este momento no existen cambios significativos durante los restantes 50 minutos. La frecuencia cardiaca promedio fue de $154 \pm 28$ latidos por minuto y la frecuencia respiratoria promedio fue de $63 \pm 33$ respiraciones por minuto durante el procedimiento. La oximetría promedio fue de $88 \pm 8 \%$, se encontró diferencia significativa sólo en el minuto 40 de la anestesia. Aparentemente el protocolo anestésico a base de tramadol, acepromacina, xilazina y ketamina es seguro a las dosis utilizadas para la enseñanza de la cirugía, en donde las FC, FR y la Ox aparentemente se comportan con estabilidad, sin embargo, se recomienda suplementar oxigeno durante la anestesia.

Palabras clave: conejo, anestesia, tramadol, cardiorrespiratorio.

\section{INTRODUCCIÓN}

Los conejos son considerados animales difíciles de anestesiar, debido a sus peculiaridades anatómicas y fisiológicas, como su proporción torácica reducida, además de ser animales fáciles de estresar. Durante la anestesia general se informo una mortalidad de $1.38 \%$ en el Reino Unido (Grint y Murison 2008), cuando se utiliza la combinación de ketamina y xilazina se incrementa a 5,8\% (Henke y col 2005) y en la enseñanza quirúrgica se reporta hasta

Aceptado: 29.08.2013.

* Calzada del Hueso 1100 Colonia Villa Quietud Delegación Coyoacán, Ciudad de México, CP 04960, México; jjperez1_1999@ yahoo.com, perivet@gmail.com un $40 \%$ de mortalidad debida a problemas anestésicos y complicaciones transoperatorias (Tapia y col 2005).

La utilización del conejo Nueva Zelanda (Oryctolagus cuniculus) como modelo para la cirugía experimental y enseñanza de la cirugía es una práctica común debido a que todavía no se han desarrollado modelos in vitro que simulen la complejidad de las respuestas de los seres vivos (Calasans-Maia y col 2009), por lo que es indispensable mejorar nuestras técnicas y eso incluye el refinamiento de nuestros procedimientos anestésicos, los cuales deben de procurar el bienestar animal, lo cual implica la utilización de al menos un analgésico que complemente a los anestésicos.

El principio de las tres " $R$ " se refiere a la reducción y remplazo de los animales utilizados para la investigación y docencia, así como al refinamiento de las técnicas utilizadas en ellos. Esto incluyen todas aquellas técnicas 
que salvaguarden el bienestar animal, un ejemplo claro es la utilización de analgésicos de manera previa y no sólo después de los procedimientos que generen dolor en los animales (Coulter y col 2011).

Existen informes sobre el uso de diferentes combinaciones de anestésicos la mayoría de ellos son utilizados solo como inductores de la anestesia, la cual, es mantenida con agentes inhalados como el isofluorano y el sevofluorano (Borkowski y Karas 1999, Lichtenberger y Ko 2007, Lennox 2008).

La anestesia fija, también ha sido descrita en conejos, esta es producida por la co-administración de fármacos por lo general la ketamina combinada con un agente sedante como: acepromacina, xilazina, diazepam o midazolam entre otros. Sin embargo, debido a la alta tasa metabólica de esta especie, la eliminación de los fármacos es muy rápida, por lo que para obtener una anestesia quirúrgica se tienen que administrar dosis elevadas y repetidas de estos fármacos (Borkowski y Karas 1999), lo cual favorece la depresión respiratoria, además, la analgesia que proporcionan estas combinaciones para cirugía mayor es inadecuada (Henke y col 2005). Son muy pocos los protocolos que incluyen un analgésico propiamente dicho. Se ha descrito que el $50 \%$ de los conejos que son sometidos a procedimientos anestésicos reciben algún tipo de analgesia en el periodo perioperatorio, el resto únicamente lo recibe en el periodo postoperatorio, siendo el fármaco más utilizado la buprenorfina y con menor frecuencia se ha reportado el uso de antiinflamatorios no esteroidales (Coulter y col 2011).

Una de las combinaciones anestésicas que se han utilizado y valorado en conejos es la de ketamina y xilazina por las propiedades amnésicas, analgésicas somáticas, disociación con el medio ambiente y la relajación muscular que provee. La duración reportada de esta combinación varía entre 25 y 40 minutos y cuando se le adiciona acepromacina a la combinación la duración se incrementa entre 45 y 79 minutos (Flecknell 2009, Lipman y col 1990), siendo una de sus principales cualidades la de mantener al conejo con respiración espontanea durante la anestesia (Yershov y col 2007).

Para complementar el protocolo anestésico a base de ketamina y xilazina con el fin de prolongar su tiempo de duración y mejorar la calidad de la analgesia en animales sanos, se realiza la adición de una combinación neuroleptoanalgesica (NLA). De esta manera se logra una anestesia general fija balanceada, la cual aportara mayor seguridad al realizar la anestesia a toxicidad dispersa, evitando así el uso de grandes dosis de anestésicos únicos (Ilkiw 1999).

Uno de los agentes más utilizados como parte de la NLA es la acepromacina, la cual es un derivado de la fenotiazina con propiedades antieméticas y anti-arrítmicas, interviene con la transmisión de dopamina en el sistema nervioso, produciendo un estado de moderada sedación sin analgesia, este efecto hace posible la disminución de los requerimientos de anestésicos inyectables e inhala- dos. La acepromacina ha sido combinada con diversos opiodes para realizar la NLA en perros dentro de ellos el tramadol (Monteiro y col 2009, Fajardo y col 2012). Éste es un analgésico sintético, derivado de la codeína con poca afinidad por los receptores opiáceos $\mu$ (Küçük y col 2005). Su potencia analgésica depende de mecanismos opioides y no opioides, este agente inhibe la recaptación de serotonina y norepinefrina logrando la modulación del dolor a nivel espinal y así el bloqueo de impulsos que llegan al cerebro (Monteiro y col 2009). La analgesia que produce cuando es administrado de manera preoperatoria en el perro, es similar a la de la morfina (Mastrocinque y Fantoni 2003). Se ha utilizado solo en el control del dolor postoperatorio en pequeños mamíferos (Lichtenberger y Ko 2007, Pereira y col 2010). En conejos mediante estudios de cromatografía líquida se ha determinado que la vida media del tramadol administrado por vía intravenosa es de una hora (Küçük y col 2005).

El objetivo del presente trabajo fue el de evaluar la estabilidad cardiorrespiratoria en conejos utilizados para enseñanza quirúrgica, los cuales fueron anestesiados con la combinación de tramadol, acepromacina, ketamina y xilazina.

\section{MATERIAL Y MÉTODOS}

Veinticinco (25) conejos (Oryctolagus cuniculus) de la raza Nueva Zelanda de ambos sexos con un peso promedio de 3,3 $\pm 0,5$ kilogramos, clínicamente sanos, provenientes de la Unidad de Producción y Experimentación en Animales de Laboratorio de la Universidad Autónoma Metropolitana Unidad Xochimilco (UAM-X), con ayuno de 5 horas de sólidos y de 1 hora de líquidos. Una vez en la sala de preparación los animales pasaron veinte minutos de adaptación previo a la medición de los valores basales (minuto "0") de las frecuencias cardiaca (FC), frecuencia respiratoria (FR) utilizando para ello un estetoscopio y un cronómetro y se obtuvieron medidas de la saturación de oxigeno de la hemoglobina mediante un oxímetro de pulso $(\mathrm{Ox})$ (Vitasign $®)^{1}$ colocando el sensor sobre los vasos centrales de la oreja.

Los conejos fueron anestesiados con una combinación de acepromacina $(0,5 \mathrm{mg} / \mathrm{kg})$, ketamina $(50 \mathrm{mg} / \mathrm{kg})$ y xilazina $(5 \mathrm{mg} / \mathrm{kg})$, mezclados en la misma jeringa y administrados por vía intramuscular en el miembro pélvico (masa muscular formada por los músculos vastos) por una sola vez, a partir de este momento los valores de FC, FR y Ox fueron obtenidos cada 10 minutos hasta el minuto 60 post-administración de la combinación anestésica. Cuando los conejos entraron en sedación profunda (8 minutos después de la inyección), se les colocó un abrebocas, se insertó un catéter vascular periférico calibre $24 \mathrm{G}(\text { Surflash } \AA)^{2}$ en la vena marginal de la oreja, se

\footnotetext{
West Medica Austria

Terumo Medical de México SA de CV.
} 
inició con el goteo de solución de cloruro de sodio $0.9 \%$ $(10 \mathrm{ml} / \mathrm{kg} / \mathrm{hr})$, al minuto 10 se administró tramadol $(5 \mathrm{mg} /$ $\mathrm{kg}$ ) por vía intravenosa.

Cinco equipos quirúrgicos conformados por 4 alumnos cada uno, inscritos en el curso de Bases de Terapéutica Quirúrgica Veterinaria (supervisados por sus instructores) durante cinco sesiones de entrenamiento se encargaron del monitoreo y control anestésico, en cada sesión de entrenamiento se les realizó una laparotomía exploratoria por la línea media abdominal, teniendo la herida quirúrgica una extensión de $15 \mathrm{~cm}$ con fines de enseñanza quirúrgica, al término todos los animales fueron eutanaciados por sobredosis de pentobarbital sódico $(50 \mathrm{mg} / \mathrm{kg})$ intravenoso.

Para valorar la profundidad anestésica se utilizaron las respuestas al estimular el pabellón auricular, que se evaluaron como negativas cuando no existe movimiento del pabellón auricular al estimularlo con la punta de un lápiz. Además se valoró la presencia o ausencia de reflejo podal, el cual se estimuló por el pinzamiento de la piel del espacio interdigital entre el tercer y cuarto dedo en los miembros torácicos con una pinza de Halsted, considerándose negativo si el conejo no retraía el miembro pinzado (Henke y col 2005, Flecknell 2009). Basado en la respuesta individual de cada conejo a los reflejos antes mencionados, solo un conejo requirió una dosis adicional de $15 \mathrm{mg}$ de Ketamina por vía intravenosa al minuto 40.

\section{ANÁLISIS ESTADÍSTICO}

El diseño del estudio se realizó en base a mediciones repetidas, se calculó la media y la desviación estándar para las FC, FR y Ox.

Se determinó mediante la prueba de Shapiro-Wilk que los datos obtenidos provenían de una muestra con distribución diferente a la normal, por lo que se utilizaron las pruebas no paramétricas de Kruskal-Wallis de una vía y la prueba pareada de Mann-Whitney, para comparar los valores de FC, FR y Ox, obtenidos entre los diferentes intervalos de tiempo a lo largo de los "60" minutos. Se consideró estadísticamente significativo un valor de $\mathrm{P}<0,05$. Se empleó para este fin el programa Palaeontological Statistics (PAST) ${ }^{3}$, ver. 1.81 .

Este estudio se encuentra aprobado por el Comité Interno para el Cuidado y Uso de los Animales de Laboratorio UAM-X.

\section{RESULTADOS Y DISCUSIÓN}

La FC presentó un valor promedio inicial en el minuto "0" de $182 \pm 43$ latidos por minuto (lpm) finalizando en $151 \pm 24 \mathrm{lpm}$ al minuto " 60 ", con promedio durante todo

\footnotetext{
Hammer Ø, DAT Harper, PD Ryan. 2001. PAST: Paleontological Statistics Software Package for Education and Data Analysis. Palaeontologia Electrónica. http://palaeo-electronica.org/2001_1/ past/issue1_01.htm, accesed february, 2012.
}

el procedimiento de $154 \pm 28 \mathrm{lpm}$. La FR promedio varió de $126 \pm 35$ respiraciones por minuto (rpm) al minuto " 0 " a $66 \pm 23 \mathrm{rpm}$ al minuto " 60 " con un promedio de $63 \pm 33$ rpm durante el procedimiento. La frecuencia cardiaca y la respiratoria tuvieron una diferencia significativa $(\mathrm{P}<0,05)$ entre el minuto " 0 " y el minuto " 10 ". No se observaron cambios significativos en el comportamiento de las FC y FR desde el minuto "10" y durante los restantes 50 minutos como se muestra en la figura 1. El promedio de la Ox al minuto " 0 " fue de $93 \pm 5 \%$ finalizando al minuto " 60 " en $85 \pm 10 \%$, siendo el promedio durante todo el procedimiento de $88 \pm 8 \%$, solo se observó una diferencia significativa en el minuto 40 de la anestesia $83 \pm 8 \%(\mathrm{P}<0,05)$.

Existen pocos estudios que valoren la estabilidad longitudinal de la FC y FR en conejos, sin embargo, son indispensables para tener rangos de referencia respecto a las frecuencias que un conejo tiene durante la anestesia. De manera puntual se ha reportado la frecuencia cardiaca en conejos Nueva Zelanda anestesiados con diferentes fármacos y vías de administración. Cuando se utilizan combinaciones de medetomidina $(0,25 \mathrm{mg} / \mathrm{kg})$ y ketamina $(15 \mathrm{mg} / \mathrm{kg}$ ) por vía intramuscular, la FC promedio es de $214 \pm 17 \mathrm{lpm}$ (Hedenqvist y col 2002). De manera adicional con la administración endovenosa de xilazina $(0,4 \mathrm{mg} /$ $\mathrm{kg}$ ) y ketamina (4 mg/kg) se reportó un promedio de $163 \pm$ 28 lpm (Baumgartner y col 2010). En el presente estudio la FC promedio del animal anestesiado fue de $151 \pm 24 \mathrm{lpm}$ al minuto "10" este valor es menor que lo reportado por Hedenqvist y col (2002), así como por Baumgartner y col (2010). Por otra parte cuando se emplea una combinación de ketamina $(35 \mathrm{mg} / \mathrm{kg})$, xilazina $(5 \mathrm{mg} / \mathrm{kg})$ y acepromacina $(0,75 \mathrm{mg} / \mathrm{kg})$ por vía intramuscular, Lipman y col (1990) observaron una disminución del 5\% en la FC durante los primeros 10 minutos después de la administración de los fármacos. En el presente estudio se observó depresión de la FC del 17\% 10 minutos posteriores a la administración de la combinación de fármacos, esta mayor depresión de la FC puede ser atribuida al incremento de la dosis de ketamina, sin embargo después de este periodo de inducción de la anestesia la FC se mantiene estable, esto puede deberse al efecto de la ketamina, la cual produce estabilidad hemodinámica como resultado de su efecto estimulante del Sistema Nervioso Simpático (Cruz y Col 2010).

Por otro lado, la combinación de acepromacina, xilazina y ketamina produce depresión del $86 \%$ en la FR durante los 10 primeros minutos después de su aplicación (Lipman y col 1990). En este estudio la depresión respiratoria fue del $48 \%$. Aunque la dosis de ketamina fue 15 mg más alta en nuestro estudio, la dosis de acepromacina fue $0,25 \mathrm{mg}$ más baja y se adicionó tramadol, este último no se reporta como depresor respiratorio (Fajardo y col 2012). Es probable que la diferencia en la depresión respiratoria reportada por Lipman y col (1990), esté dada por el aumento en la dosis de acepromacina utilizada, por otro lado la diferencia estadística en la FR al minuto 40 se puede deber a la dosis adicional de ketamina en un conejo. 


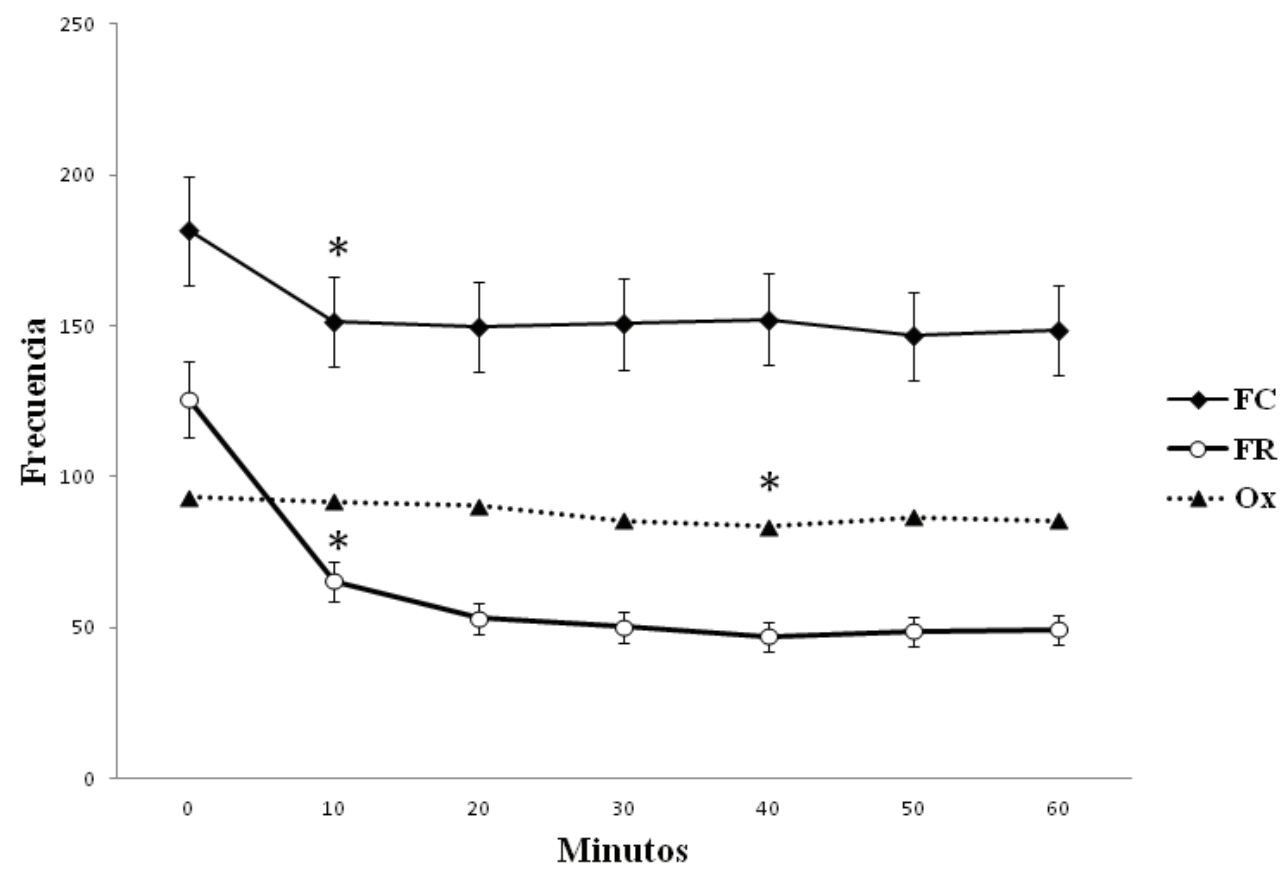

Figura 1. Media y desviación estándar de la frecuencia cardiaca (FC) expresada en latidos por minuto, frecuencia respiratoria (FR) expresada en respiraciones por minuto y porcentaje de saturación de oxigeno (Ox) de los conejos ( $\mathrm{n}=25)$ durante los 60 minutos de anestesia. *Diferencia estadísticamente significativa $(\mathrm{P}<0,05)$.

Average and standard derivation of heart rate $(\mathrm{FC})$ in beats per minute, respiratory rate $(\mathrm{FR})$ expressed in breaths per minute and percentage of oxygen saturation $(\mathrm{Ox})$ of rabbits $(\mathrm{n}=25)$ during 60 minutes anesthesia. * Statistically significant difference $(\mathrm{P}<0.05)$.

Este estudio reportó una FR promedio de $63 \pm 33$ rpm, mientras que la administración de ketamina y medetomidina produjo en promedio un valor de la FR de $49 \pm 7$ respiraciones por minuto la cual disminuyó a $31 \pm 5 \mathrm{lpm}$ al adicionarle butorfanol como analgésico, (Hedenqvist y col 2002). Aunque ambos protocolos incluyen un analgésico opioide, al comparar los cambios en la profundidad de la sedación en perros bajo el efecto de acepromacina, cuando se les administran analgésicos opioides por vía intravenosa como el butorfanol y el tramadol, este último tiene menos efectos depresores sobre la respiración (Monteiro y col 2009), lo que en parte explica las diferencias en las FR promedio.

En el caso de la Ox los valores se mantuvieron en niveles de saturación críticos para animales de laboratorio anestesiados (Flecknell 2009, Hedenqvist y col 2002). Aunque no hay reportes de oximetría en el conejo, Yershov y col (2007) mencionan que por las características anatómicas del conejo la oxigenación adecuada durante la anestesia es difícil. En nuestro estudio el único manejo realizado para mejorar la ventilación fue la utilización de abrebocas, lo cual demuestra que no fue suficiente para mantener los niveles de saturación de oxigeno dentro de valores seguros (>95\%), los resultados hacen necesario recomendar mejor control de la vía aérea, así como suplementar con oxígeno cuando se utilice esta combinación anestésica en conejos.
La combinación sólo fue evaluada clínicamente en conejos sanos de la raza Nueva Zelanda, sin embargo, una observación clínica por parte de los autores, fue que una dosis del $80 \%$ del total de la utilizada en conejo Nueva Zelanda fue suficiente para realizar una orquiectomía en conejo mestizo. Es necesario, verificar su funcionalidad clínica en otras razas o animales con algún tipo de enfermedad.

El protocolo anestésico a base de tramadol, acepromacina, xilazina y ketamina generó una menor depresión respiratoria y mayor depresión cardiaca que lo reportado de manera previa, este permitió la realización de una cirugía con fines de enseñanza, manteniendo la FC, FR estables a lo largo del tiempo y la Ox estable pero por debajo del rango permisible. Es necesario evaluar el protocolo anestésico suplementando oxígeno y mediante otros parámetros como presión arterial, electrocardiografía, gasometría, así como su efecto a nivel hepático y renal con la finalidad de verificar su seguridad.

\section{REFERENCIAS}

Baumgartner C, M Bollerhey, J Ebner, L Laacke-Singer, T Schuster, W Erhardt. 2010. Effects of ketamine-xylazine intravenous bolus injection on cardiovascular function in rabbits. Can J Vet Res 74, 200-208. 
Borkowski R, AZ Karas. 1999. Sedation and Anesthesia of Pet Rabbits. Clin Tech Small Anim Pract 14, 44-49.

Calasans-Maia MD, ML Monteiro, F Oliveira Áscoli, JM Granjeiro. 2009. The rabbit as an animal model for experimental surgery. Acta Cir Bras 24, 325-328.

Coulter CA, PA Flecknell, MC Leach, CA Richardson. 2011. Reported analgesic administration to rabbits undergoing experimental surgical procedures. BMC Vet Res 7, 12.

Cruz FS, AB Carregaro, AG Raiser, M Zimmerman, R Lukarsewski, RP Steffen. 2010. Total intravenous anesthesia with propofol and $\mathrm{S}(+)$-ketamine in rabbits. Vet Anaesth Analg 37, 116-122.

Fajardo MA, MA Lesmes, LA Cardona. 2012. Evaluación del efecto analgésico postoperatorio de infusiones intraoperatorias de tramadol y tramadol/lidocaína/ketamina en comparación con morfina/lidocaína/ketamina en hembras caninas sometidas a ovariohisterectomía. Arch Med Vet 44, 145-153

Flecknell P. 2009. Laboratory Animal Anaesthesia. $3^{\text {rd }}$ ed. Academic Press, San Diego, USA.

Grint NJ, PJ Murison. 2008. A comparison of ketamine-midazolam and ketamine-medetomidine combinations for induction of anaesthesia in rabbits. Vet Anaesth Analg 35, 113-121.

Hedenqvist P, HE Orry ,JV Roughany, LM Antunesy, PA Flecknell. 2002. Anaesthesia with ketamine/medetomidine in the rabbit: influence of route of administration and the effect of combination with butorphanol. Vet Anaesth Analg 29, 14-19

Henke J, S Astner, T Brill, B Eissner, R Busch, W Erhardt. 2005. Comparative study of three intramuscular anaesthetic combinations (medetomidine/ketamine, medetomidine/fentanyl/midazolam and xylazine/ketamine) in rabbits. Vet Anaesth Analg 32, 261-270.

Ilkiw JE. 1999. Balanced Anesthetic Techniques in Dogs and
Cats. Clin Tech Small Anim Pract 14, 27-37.

Küçük A, Y Kadioğlu, F Çelebi. 2005. Investigation of the pharmacokinetics of tramadol in rabbit plasma by a High-Performance Liquid Chromatography-Diode array detector method using liquid-liquid extraction. J Cromatography $B$ 816, 203-208.

Lennox AM. 2008. Clinical Technique: Small exotic companion mammal dentistry-anesthetic considerations. J Exotic Pet Med 17, 102-106.

Lichtenberger M, J Ko. 2007. Anesthesia and analgesia for small mammals and birds. Vet Clin Exot Anim 10, 293315.

Lipman NS, RP Marini, SE Erdman. 1990. A comparison of Ketamine/Xilazine and Ketamine/xilazine/Acepromacine anesthesia in the rabbit. Lab Anim Sci 40, 395-398.

Mastrocinque S, DT Fantoni. 2003. A comparison of preoperative tramadol and morphine for the control of early postoperative pain in canine ovariohysterectomy. Vet Anaesth Analg 30, 220-228.

Monteiro ER, AR Junior, HM Assis, D Campagnol, JG Quitzan. 2009. Comparative study on the sedative effects of morphine, methadone, butorphanol or tramadol, in combination with acepromazine, in dogs. Vet Anaesth Analg 36, 25-33.

Pereira BR, TMR Maciel, MF Schons, MAL Letti, A Jr da Rosa, AG Nunes, R da Silva. 2010. Anesthetic protocol for videolaparoscopic surgery in rabbits. Acta Cir Bras 25, 121-125

Tapia JJ, MB León, GC Baños, LF García. 2005. Apendicectomía por laparoscopia en el conejo como modelo experimental. Rev Fac Med Univ Nac Auton Mex 48, 232-235.

Yershov AL, S Bryan, BS Jordan, M James, JM Fudge, MA Dubick. 2007. Influence of the mode of ventilation on ketamine/xylazine requirements in rabbits. Vet Anaesth Analg 34, 157-163. 
